\title{
Split Ring Resonators With Tapered Strip Width for Wider Bandwidth and Enhanced Resonance
}

\author{
Ali K. Horestani, Student Member, IEEE, Christophe Fumeaux, Senior Member, IEEE, \\ Said F. Al-Sarawi, Member, IEEE, and Derek Abbott, Fellow, IEEE
}

\begin{abstract}
This article presents a modified edge-coupled split ring resonator (SRR). The proposed SRR is composed of two concentric metallic rings with a nonuniform strip width, which is tapered based on the current and voltage profile in the SRR structure. In contrast to other SRR miniaturization methods, which are based on increasing the equivalent capacitance of the SRR, the proposed SRR benefits from both increased capacitance and inductance to preserve the strength and bandwidth of the resonance. It is also shown that compared to a uniform SRR, a tapered SRR with the same electrical size provides a stronger resonance with $84 \%$ wider bandwidth, as desired in wideband filter design. The theory and simulation results are validated through measurement.
\end{abstract}

Index Terms-Metamaterials, split ring resonator (SRR).

\section{INTRODUCTION}

$\mathbf{G}$ ENERALLY there are two main methods for the realization of planar metamaterials with negative permeability. In the first method a composite right/left-handed transmission line is achieved by periodically loading a conventional transmission line (TL) with series capacitors and shunt inductors [1]. The second method is based on loading a conventional TL by sub-wavelength resonators, such as split ring resonators (SRRs) [2], [3]. In spite of the advantages of the metamaterial structures based on the SRRs, such as easier fabrication and application in bulk metamaterial media, SRRs are more lossy and have a narrower bandwidth [4].

Many investigations have aimed at achieving SRRs with small electrical size, wide bandwidth and strong resonance [5]-[7]. It has been shown using equivalent circuit models for SRRs that the fractional area occupied by the interior of the resonator's ring within the unit cell can be enlarged in order to achieve a relatively wider bandwidth resonance [6], [8]. However, this is in conflict with the miniaturization of the structure, which is required for compact filters or to realize an effective medium. In order to create miniaturized left-handed media, other sub-wavelength resonators have been proposed. For instance, compared to the edge-coupled SRR proposed by Pendry [2], the broad-side coupled split ring resonator (BC-SRR) [6] benefits from smaller electrical size. However,

Manuscript received June 11, 2012; accepted July 22, 2012. Date of publication August 14, 2012; date of current version August 30, 2012.

Ali K. Horestani is with the School of Electrical and Electronic Engineering, The University of Adelaide, Adelaide, SA 5005, Australia and also with the Ministry of Science, Research and Technology, Tehran, Iran (e-mail: akarami@eleceng.adelaide.edu.au).

C. Fumeaux, S. Al-Sarawi, and D. Abbott are with the School of Electrical and Electronic Engineering, The University of Adelaide, Adelaide, SA 5005, Australia.

Color versions of one or more of the figures in this paper are available online at http://ieeexplore.ieee.org.

Digital Object Identifier 10.1109/LMWC.2012.2211341 due to the increased equivalent capacitance, the resonator has a narrower bandwidth. Furthermore, the fabrication of the structure is technologically more complex because of the need for a multilayer substrate. Other structures such as spiral resonators [5] are compact and uniplanar, but compared to the edge-coupled SRRs, they are less efficient and provide weak resonances. Alternatively, methods, such as loading a single split ring resonator with a semi-lumped $L C$ resonator [4] have been used for controlling the resonance bandwidth. However, this method results in narrower bandwidths with weaker resonances, and since it is based on single split ring, the structure has a relatively larger electrical size.

In this letter a modified edge-coupled SRR with reduced electrical size is proposed. The proposed SRR has a strip width tapered in steps determined by considering the distribution of the current and voltage in the SRR structure. It is also shown that compared to the conventional SRRs, a tapered SRR with the same electrical size provides a wider bandwidth and a stronger resonance.

\section{Resonance Characteristics of Split RING RESONATOR COUPLED TO A TL}

Fig. 1(a) depicts a simple circuit model for a unit cell of a TL loaded with an SRR, where $L_{r}$ and $C_{r}$ are equivalent inductance and capacitance of the SRR, respectively, and $R_{r}$ models the SRR's losses. The magnetic coupling between the SRR and the loaded TL, $M$, can be approximated by $M=L_{r} \cdot \alpha F$ [8], where $F$ is the fraction of the cell area occupied by the SRR and $\alpha$ is a fitting parameter. Near resonance, the model can be further simplified into the circuit model of Fig. 1(b) [4], where $L=$ $M^{2} / L_{r}, C=L_{r} /\left(\omega_{0}^{2} M^{2}\right), R=\omega_{0} Q_{0} M^{2} / L_{r}$. The resonant frequency can be obtained from

$$
\omega_{0}=\frac{1}{\sqrt{L C}}=\frac{1}{\sqrt{L_{r} C_{r}}} .
$$

The coupling factor of the TL with a loading resonator is defined as the ratio of the resonator resistance at the resonance frequency to the sum of the external resistances which are observed from the resonator ports [9]. Using the simplified model of the SRR-loaded TL, Fig. 1(b), the coupling factor $\beta$ can be expressed in terms of the circuit model parameters as

$$
\beta=\frac{R}{R_{\text {ext }}}=\frac{R}{2 Z_{0}}=\frac{1}{2 Z_{0} R_{r}} \cdot \frac{L_{r}}{C_{r}} \cdot(\alpha F)^{2} .
$$

The coupling factor can also be obtained from simulated or measured scattering parameters as [9]

$$
\beta=\frac{\left|S_{11_{0}}\right|}{\left|S_{21_{0}}\right|}
$$




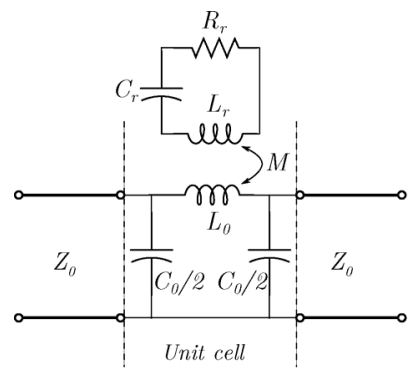

(a)

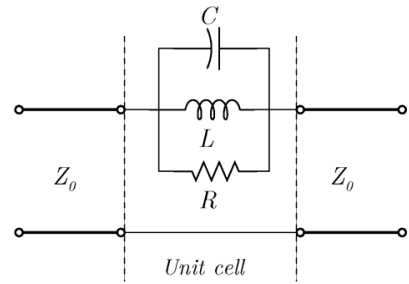

(b)
Fig. 1. Equivalent circuit model [4] of an SRR loaded transmission line, including the SRR loss (a), and the simplified equivalent circuit model (b).

where $S_{11_{0}}$ and $S_{21_{0}}$ are the reflection and the transmission coefficients of the structure at the resonant frequency $f_{0}$, respectively. It is worth emphasizing that based on (3) a high coupling factor translates into a high ratio of $\left|S_{11}\right|$ and $\left|S_{21}\right|$ at resonance, which means a stronger (i.e., higher $Q$ ) resonance. Thus, even though based on (1) the resonant frequency and electrical size of the SRR can be reduced by increasing either $C_{r}$ or $L_{r}$, (2) and (3) show that decreasing the resonant frequency by increasing the SRR's equivalent capacitance results in a smaller coupling factor and consequently a weaker resonance. For instance, in [10], smaller electrical size, which is achieved by using interdigital capacitor, leads to a weak resonance. In contrast, (2) shows that increasing the SRR's equivalent inductance not only reduces the electrical size of the particle but also increases the coupling coefficient and results in a stronger resonance.

By using the following simplified equation of the loaded bandwidth for the structure [4],

$$
B W_{L} \approx \sqrt{\frac{L_{r}}{C_{r}}} \cdot \frac{(\alpha F)^{2}}{2 Z_{0}}
$$

it is also clear that while increasing the $C_{r}$ reduces the resonance bandwidth, increasing the SRR's equivalent inductance $L_{r}$ results in a resonance with a wider bandwidth, which is required in applications such as wideband filter design.

\section{TAPERED SPLIT RING RESONATOR}

High level of miniaturization, while preserving the strength and bandwidth of the resonance, can be achieved by simultaneously increasing the equivalent capacitance and inductance of the SRR. However, there is a conflict between increasing the inductance and capacitance of the SRR, i.e., increasing the equivalent inductance of the SRR by using widely spaced narrow rings results in a smaller equivalent capacitance, and vice versa.

In order to simultaneously increase the equivalent inductance and capacitance of an SRR structure, an SRR with tapered strips width is proposed here. A concept of tapering a TL based on the voltage and current distribution along the TL to improve the TL's quality factor has been used for different applications, especially for obtaining high quality factor resonators [11], [12]. Since there is a similar current distribution along the SRR rings [5], as schematically shown with arrows in Fig. 2(a), a similar tapering strategy can be used to achieve an SRR with simultaneously increased equivalent inductance and capacitance. In fact, as shown in Fig. 2(b), an SRR with a lower resonant frequency can be realized by narrowing the SRR's rings where the current

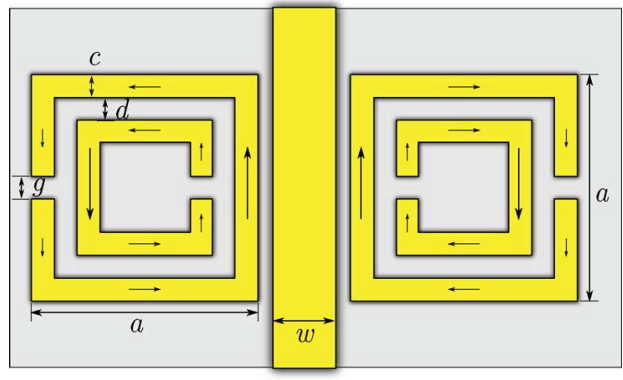

(a)

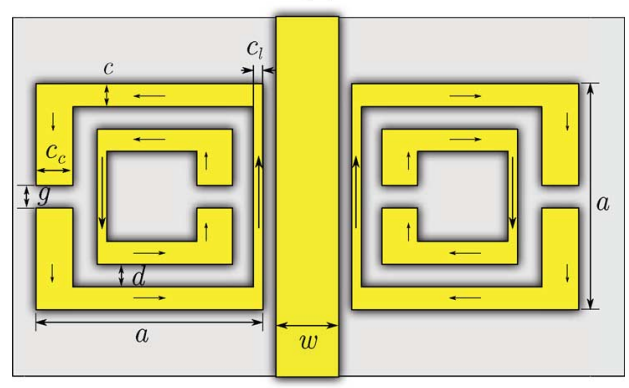

(b)

Fig. 2. Microstrip line loaded with a pair of uniform SRRs (a) and tapered SRRs (b). The arrows schematically depict the relative current distribution in different segments of the SRRs' rings.

is maximal, i.e., in the middle of the rings, and widening the rings where voltage is maximal, i.e., close to the rings' splits.

To verify the theory, TLs loaded with two types of tapered SRRs are simulated and compared to a uniform SRR loaded TL. In all the cases, SRRs with outer edge length $a=10 \mathrm{~mm}$ are coupled to a $50 \Omega$ microstrip line with width $w=2.3 \mathrm{~mm}$ on a $0.78 \mathrm{~mm}$ thick Duroid substrate with relative permittivity $\epsilon_{r}=2.2$ and loss tangent $\tan (\delta)=0.0009$. For the uniform SRRs, $c=1 \mathrm{~mm}, g=0.5 \mathrm{~mm}$, and $d$ is set to be $0.2 \mathrm{~mm}$. The tapered SRRs have the same dimensions as the uniform SRRs, however, the ring widths are narrowed in their middle segment to $c_{l}=0.2 \mathrm{~mm}$ and they are widened on each side of the split to $c_{c}=1.5 \mathrm{~mm}$. Note that as shown in Fig. 2, for the sake of simplicity, square-shaped piecewise tapered SRRs are used.

Fig. 3 compares the simulated transmission coefficient of the structure based on the tapered SRRs (black line) to that of the structure based on the uniform SRRs (blue line). As predicted by the theory, the figure shows that by adapting the segment width of the SRRs, the resonant frequency is reduced by about $18 \%$ from $2.88 \mathrm{GHz}$ for the uniform SRRs to $2.35 \mathrm{GHz}$ in the case of tapered SRRs, which translates into $33 \%$ reduction in the SRRs' electrical area. Furthermore, the fractional bandwidth is increased slightly from $1.5 \%$ to $1.8 \%$.

Fig. 3 also shows the simulated transmission coefficient of a tapered SRR with increased gap size $g=3.6 \mathrm{~mm}$, and inter-ring spacing $d=1 \mathrm{~mm}$ (red line). Note that while tapering the SRR increases the equivalent inductance of the SRR, increasing the gap and inter-ring spacing decreases the equivalent capacitance of the SRR. Thus, the tapered SRR can be designed with the same resonant frequency as the uniform SRR. However, compared to the uniform SRR, the tapered SRR benefits from a higher $L_{r}$ to $C_{r}$ ratio. As predicted from (2), (3) and (4), the simulation results show that compared to the uniform SRR, the 


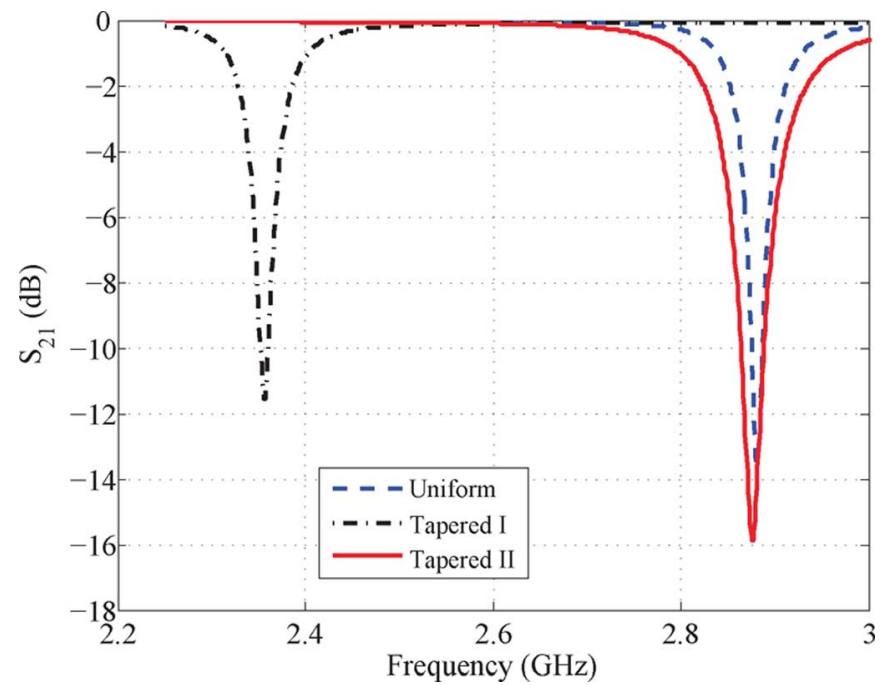

Fig. 3. Simulated transmission coefficients of TLs loaded with uniform SRRs and two types of tapered SRRs.

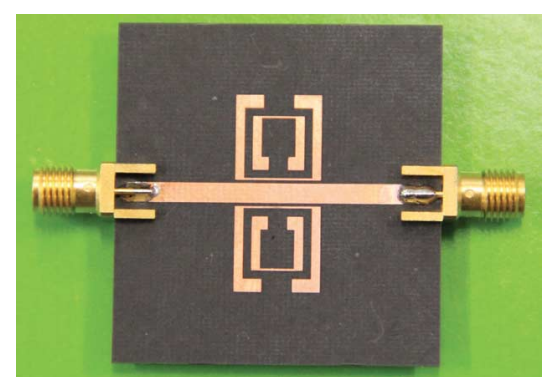

Fig. 4. Photograph of a fabricated sample of microstrip line loaded with a pair of tapered SRRs.

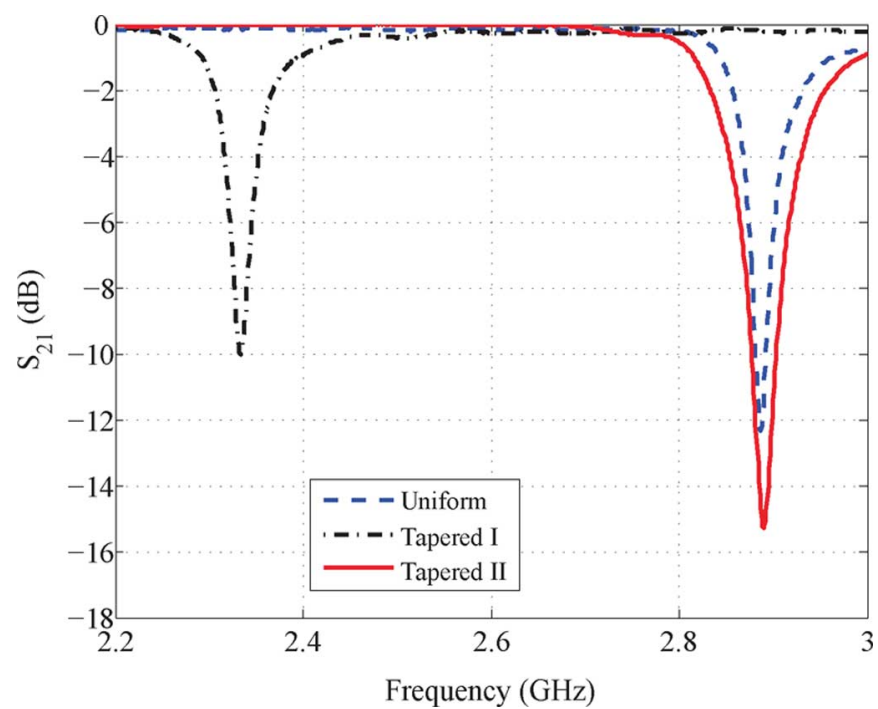

Fig. 5. Measured transmission coefficients of TLs loaded with uniform SRRs and two types of tapered SRRs.

tapered SRR exhibits a stronger resonance and has a fractional bandwidth of $2.8 \%$, i.e., $84 \%$ wider than the uniform one.

\section{EXPERIMENTAL RESULTS}

In order to validate the theory and simulation results, microstrip lines loaded with uniform and tapered SRRs have been fabricated and measured. Fig. 4 depicts the top view of one of the fabricated samples with tapered SRRs. The material used and the dimensions of the prototypes are same as the simulated structures given in Section III.

Fig. 5 depicts the measured transmission coefficient of the TLs loaded with uniform SRRs and two types of the tapered SRRs with different resonant frequencies (corresponding to the simulated structures of the previous section). The graph shows good agreement between the measured and simulated results. This agreement validates the theoretical concept of tapering SRRs for miniaturization, while improving the strength, and widening the bandwidth of the resonance. It is worth mentioning that the same principle can be applied to SRR arrays for the realization of bulk metamaterials or frequency-selective surfaces.

\section{CONCLUSION}

This work has presented a modified edge-coupled SRR with tapered strip width, which has been developed based on the current and voltage distribution in the SRR structure. The modification enabled us to reduce the SRR electrical area by $33 \%$. More importantly, it has been shown that the proposed structure with the same electrical size as a uniform SRR has a stronger resonance with $84 \%$ wider fractional bandwidth. This strong and wideband resonance is required in many applications such as a wideband filter design.

\section{REFERENCES}

[1] A. Lai, C. Caloz, and T. Itoh, "Composite right/left-handed transmission line metamaterials," Microwave Magazine, IEEE, vol. 5, no. 3, pp. 34-50, 2004.

[2] J. Pendry, A. Holden, D. Robbins, and W. Stewart, "Magnetism from conductors and enhanced nonlinear phenomena," IEEE Trans. Microw. Theory Tech., vol. 47, no. 11, pp. 2075-2084, Nov. 1999.

[3] D. Smith, W. Padilla, D. Vier, S. Nemat-Nasser, and S. Schultz, "Composite medium with simultaneously negative permeability and permittivity," Phys. Rev. Lett., vol. 84, no. 18, pp. 4184-4187, 2000.

[4] X. Lin and T. Cui, "Controlling the bandwidth of split ring resonators," IEEE Microw. Wireless Comp. Lett., vol. 18, no. 4, pp. 245-247, Apr. 2008.

[5] J. Baena, R. Marques, F. Medina, and J. Martel, "Artificial magnetic metamaterial design by using spiral resonators," Phys. Rev. B, vol. 69, no. 1, 2004, Article No. 014402.

[6] R. Marqués, F. Medina, and R. Rafii-El-Idrissi, "Role of bianisotropy in negative permeability and left-handed metamaterials," Phys. Rev. B, vol. 65, no. 14, 2002, Article no. 144440.

[7] T. Hao, C. Stevens, and D. Edwards, "Optimisation of metamaterials by Q factor,” Electron. Lett., vol. 41, no. 11, pp. 653-654, 2005.

[8] J. Baena et al., "Equivalent-circuit models for split-ring resonators and complementary split-ring resonators coupled to planar transmission lines," IEEE Trans. Microw. Theory Tech., vol. 53, no. 4, pp. 1451-1461, Apr. 2005.

[9] A. Khanna and Y. Garault, "Determination of loaded, unloaded, and external quality factors of a dielectric resonator coupled to a microstrip line," IEEE Trans. Microw. Theory Tech., vol. MTT-31, no. 3, pp. 261-264, Mar. 1983.

[10] W. Withayachumnankul, C. Fumeaux, and D. Abbott, "Compact electric- $L C$ resonators for metamaterials," Optics Express, vol. 18, no. 25, pp. 25912-25921, 2010.

[11] C. Marcu and A. Niknejad, "A $60 \mathrm{GHz}$ high-Q tapered transmission line resonator in $90 \mathrm{~nm}$ CMOS," in IEEE MTT-S Int.Dig., 2008, pp. $775-778$.

[12] A. K. Horestani, A. Mehdizadeh, S. Al-Sarawi, C. Fumeaux, and D. Abbott, "Quality factor optimization process of a tapered slow-wave coplanar strips resonator in CMOS technology," in Proc. Asia-Pacific Microw. Conf. (APMC'11), Dec. 2011, pp. 45-48. 